\title{
Concurrent and construct validity of Mediterranean diet scores as assessed by an FFQ
}

\author{
Alejandra A Benítez-Arciniega ${ }^{1,2,3}$, Michelle A Mendez ${ }^{4,5}$, Jose M Baena-Díez ${ }^{6}$, \\ Maria-Asunción Rovira Martori ${ }^{1,3}$, Cristina Soler ${ }^{7}$, Jaume Marrugat ${ }^{3,5,7}$, \\ Maria-Isabel Covas ${ }^{1,2,3}$, Hector Sanz ${ }^{7}$, Alba Llopis ${ }^{1}$ and Helmut Schröder 1,2,3, *, \\ on behalf of the REGICOR and HERMES investigators $†$ \\ ${ }^{1}$ Cardiovascular Risk and Nutrition Research Group (CARIN-ULEC), IMIM-Hospital del Mar Barcelona, Biomedical \\ Research Park (Parc de Recerca Biomèdica de Barcelona - PRBB), c/Doctor Aiguader 88, 08003 Barcelona, Spain: \\ ${ }^{2}$ CIBER Physiopathology of Obesity and Nutrition (CIBEROBN), Instituto de Salud Carlos III, Madrid, Spain: \\ ${ }^{3}$ Program of Research in Inflammatory and Cardiovascular Disorders, IMIM-Hospital del Mar, Barcelona, Spain: \\ ${ }^{4}$ Center for Research in Environmental Epidemiology (CREAL), Biomedical Research Park (Parc de Recerca Biomèdica \\ de Barcelona - PRBB), Barcelona, Spain: ${ }^{5}$ CIBER Epidemiology and Public Health (CIBERESP), Instituto de Salud \\ Carlos III, Madrid, Spain: ${ }^{6}$ Centro de Salud La Marina, IDIAP Jordi Gol, Barcelona, Spain: ${ }^{7}$ Cardiovascular \\ Epidemiology and Genetics Research Group (EGEC-ULEC), IMIM-Hospital del Mar, Barcelona, Spain
}

Submitted 10 November 2010: Accepted 26 April 2011: First published online 11 July 2011

\begin{abstract}
Objective: The aim of the present study was to assess the concurrent and construct validity of two diet-quality indices, a modified Mediterranean diet score (mMDS) and a Mediterranean-like diet score (MLDS) additionally incorporating unhealthy food choices, as determined by an FFQ.

Design: A validation study assessing FFQ intake estimates compared with ten or more unannounced $24 \mathrm{~h}$ recalls. Pearson's correlation coefficients, intraclass correlation coefficients (ICC), Bland-Altman plots and the limits of agreement method were used to assess the between-method agreement of scores. Construct validity was shown using associations between nutrient intakes derived from multiple $24 \mathrm{~h}$ recalls and the mMDS and MLDS derived from the FFQ.

Setting: Gerona, Spain.

Subjects: A total of 107 consecutively selected participants from a populationbased cross-sectional survey.

Results: Pearson's correlations for the energy-adjusted mMDS and MLDS compared with multiple recalls were $0 \cdot 48$ and $0 \cdot 62$, respectively. The average FFQ energy-adjusted mMDS and MLDS were $102 \%$ and $98 \%$ of the recall-based mMDS and MLDS estimates, respectively. The FFQ under- and overestimated dietary recall estimates of the energy-adjusted MLDS by $28 \%$ and $25 \%$, respectively, with slightly wider boundaries for the mMDS (31\% and 34\%). The ICC, which assesses absolute agreement, was similar to Pearson's correlations (mMDS $=0 \cdot 48$ and MLDS $=0 \cdot 61$ ). The mean differences between methods were similar across the range of average ratings for both scores, indicating the absence of bias. The FFQderived mMDS and MLDS correlated in the anticipated directions with intakes of eleven $(73 \cdot 3 \%)$ and thirteen of fifteen nutrients $(86 \cdot 7 \%)$, respectively.

Conclusions: The FFQ provides valid estimates of diet quality as assessed by the mMDS and MLDS.
\end{abstract}

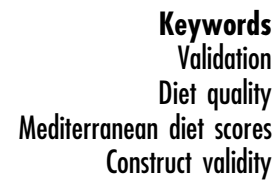

Keywords

Validation diet scores Construct validity
Dietary scores are composite constructs of dietary components used to estimate overall dietary quality ${ }^{(1-3)}$. These predefined combinations of foods and/or nutrients provide single operative variables and are considered valuable tools for the analysis of associations between

$\dagger$ A full roster of REGICOR investigators and collaborators can be found at www.REGICOR.org/regicor.inv diet quality and health outcomes. Index summary scores, such as the Healthy Eating Index, Diet Quality Index or the Mediterranean diet score (MDS), are based on interpretation of current dietary guidance and on dietary recommendations ${ }^{(4,5)}$. The traditional MDS was proposed by Trichopoulou et al. ${ }^{(6)}$ in 1995 as a tool to assess the degree of adherence to the traditional Mediterranean diet. Assessing adherence to the Mediterranean diet has 
received increasing attention during recent years because of the beneficial effect of this dietary pattern on various aspects of human health ${ }^{(5-9)}$.

Large epidemiological studies have relied primarily on the FFQ approach to estimate habitual individual dietary intake $^{(10)}$. However, concerns have been raised recently about the ability of participants to provide valid reports of dietary behaviour using these tools, particularly given the demonstration of disparities in diet-disease associations derived from an FFQ compared with alternative instruments ${ }^{(11,12)}$. Therefore, assessments of the validity of data derived from an FFQ, or the degree to which the instrument really measures what it attempts to measure, are essential.

Most FFQ validation studies have focused on comparing intake estimates using the instrument with those obtained using a reference method to determine whether it reasonably ranks subjects on the basis of their reported intakes of individual nutrients and/or food groups. However, it is impossible to determine from these data whether the FFQ accurately ranks participants against a composite score composed of multiple nutrients and/or foods, such as the MDS. We are not aware of studies assessing the validity of the MDS across dietary assessment methods. The objective of the present study was to estimate the concurrent and construct validity of two variants of MDS assessment in a subpopulation of a representative Spanish population compared with a series of ten or more unannounced $24 \mathrm{~h}$ recalls.

\section{Methods}

\section{Subjects}

Participants were selected from a population-based crosssectional survey conducted in Spain in 2005 (REGICOR study) ${ }^{(13)} ; 150$ men and women were selected consecutively. Forty-three participants with incomplete records were excluded; complete dietary data from an FFQ and from at least ten completed $24 \mathrm{~h}$ recalls were available for 107 individuals. Participants with complete data did not differ from the initial validation sample with respect to variables potentially related to diet quality, including age, gender and BMI (not shown). The project was approved by the local ethics committee (Comités Éticos de Investigación Clínica Instituto Municipal de Asistencia Sanitaria, Barcelona, Spain).

\section{Dietary assessment}

\section{FFQ}

Food consumption was estimated using a validated ${ }^{(14)}$ FFQ administered by a trained interviewer. In a 166-item food list including alcoholic and non-alcoholic beverages (typical foods in north-eastern Spain), participants indicated their usual consumption and chose from ten frequency categories, ranging from never or $<1$ time/month to $\geq 6$ times/d. Food items were listed under fourteen food groups: milk and dairy products, cereals and grain products, vegetables, legumes, sausages, oils and fats, eggs, meat and fish, fast food, canned products, fruit, nuts, sweets and desserts, and others (salt and sugar), as well as alcoholic and non-alcoholic beverages.

\section{Multiple $24 \mathrm{~b}$ recalls}

Twelve unannounced $24 \mathrm{~h}$ dietary recalls were collected by telephone over a 12 -month period by a trained interviewer. At least ten completed recalls were required for inclusion in the analysis. Dietary recalls were conducted on non-consecutive days, including at least five weekdays and one weekend day. Food intake data recorded during the $24 \mathrm{~h}$ recalls were grouped into the food-based dietary components of the FFQ for analysis.

\section{Calculation of dietary quality scores}

The modified Mediterranean diet score. The modified MDS (mMDS) was calculated according to the tertile distribution of food consumption, with the exception of red wine ${ }^{(15,16)}$. For cereals, fruit, vegetables, legumes, fish, olive oil and nuts, the lowest tertile was coded as 1, medium as 2 and the highest as 3. For meat (including poultry and sausages) and dairy products, the score was inverted, with the highest tertile coded as 1 and the lowest as 3. Moderate red wine consumption (up to $20 \mathrm{~g}$ ) was included as a favourable component in the MDS, with a score of 3. Exceeding this upper limit or reporting no red wine consumption was coded as 0 . The resulting score ranged from 10 to 30 .

The Mediterranean-like dietary score. The Mediterraneanlike dietary score (MLDS) was constructed by adding three food groups to the ten components of the mMDS: sugarsweetened carbonated beverages and added sugars; pastries; and fast food. These food groups were scored inversely. In addition, we omitted high-fat dairy products as a negative component and instead included low-fat dairy products as a beneficial food group. We also excluded poultry and rabbit from the meat and sausage food group. The resulting scores ranged from 13 to 39.

\section{Other variables}

Measurements of demographic, socio-economic and lifestyle variables including smoking habits and alcohol consumption were obtained through structured standard questionnaires administered by trained personnel.

\section{Statistical analyses}

Differences in continuous variables were compared by the Student $t$ test or the Mann-Whitney $U$ test (for nonnormally distributed variables). Categorical variables were tested using the $\chi^{2}$ goodness-of-fit test.

Spearman's correlation coefficients and cross-classification were used to assess the capability of the FFQ to rank participants according to their food group intake or on the basis of scores obtained on the Mediterranean diet indices. Estimated intakes of individual food groups were 
also compared across instruments. Cross-classification was carried out using contingency tables of tertile distribution of the FFQ compared with the $24 \mathrm{~h}$ recall-derived mMDS and MLDS. The proportion of participants correctly categorized (same tertile) and grossly misclassified (opposite tertiles) was calculated.

The mMDS and MLDS were normally distributed. Therefore, relative agreement of the MMDS and MLDS was assessed by calculating Pearson's product-moment correlation coefficients to compare the $24 \mathrm{~h}$ recall scores (reference method) with the participants' scores on the FFQ (test method).

Two measures might be highly correlated; yet, there could be substantial differences in the two measurements across their range of values. For this reason we additionally analysed absolute agreement between two measurements by the intraclass correlation coefficient (ICC) and the Bland-Altman method ${ }^{(17)}$. The Bland-Altman analysis determines the average agreement between two methods by calculating the mean of their differences. The 95\% limits of agreement (LOA) provide an interval within which 95\% of these differences are expected to fall. Agreement between the MDS obtained from the FFQ and those obtained from $24 \mathrm{~h}$ recalls was depicted in Bland-Altman plots. A mean agreement of $100 \%$ signified complete agreement between the methods. An LOA between 50\% and $200 \%$ was considered reasonable ${ }^{(18)}$.

A one-sample $t$ test was used to determine the significance of differences between scores derived from the FFQ and those derived from $24 \mathrm{~h}$ recalls.

In addition, we analysed possible variations in the level of agreement between methods. Proportional bias indicates that the disparity between test and reference methods (i.e. the mean difference) varies significantly, depending on the magnitude of the mean ratings of dietary indices. For this purpose we performed linear regression analysis, with the mean instrument differences of the MMDS and MLDS constituting the dependent variable and the mean score for the corresponding mMDS and MLDS obtained by the test (FFQ) and reference methods ( $24 \mathrm{~h}$ recalls) constituting the independent variable.
Finally, to assess construct validity, general linear modelling was used to estimate associations between nutrient intakes (dependent variables) derived from $24 \mathrm{~h}$ recalls and from the tertile distribution of the MMDS and MLDS calculated from the FFQ (independent variable). Linear trends were tested by including the categorized variable (tertile distribution of the scores) as continuous in this model, and the $P$ value for linear trend was calculated using polynomial contrast for continuous variables. The Statistical Package for the Social Sciences statistical software package version $13 \cdot 0$ (SPSS Inc., Chicago, IL, USA) was used for all statistical analyses. Differences were considered significant if $P$ was $<0 \cdot 05$.

\section{Results}

With the exception of education, participants in the validation study did not differ significantly from the rest of the sample (Table 1). Spearman's correlation coefficients for food group intakes estimated from the FFQ and $24 \mathrm{~h}$ recalls ranged from $0 \cdot 19$ to $0 \cdot 69$, and were moderate on average (Table 2). We cross-classified food groups into tertiles to evaluate the ability of FFQ and $24 \mathrm{~h}$ recalls to rank participants on the basis of categories of intake. On average, $48.4 \%$ of the individuals were grouped into the same tertile on both instruments (ranging from 33.6\% congruence for meat to $73 \cdot 8 \%$ for red wine). Mean gross misclassification (the percentage of individuals in opposite tertiles for the same food item using the two instruments) for food groups was $13 \cdot 2 \%$, with fish being the most frequently misclassified food group (Table 2 ).

Pearson's correlation coefficients between the scores derived from the FFQ and $24 \mathrm{~h}$ recalls were moderate and good for the mMDS and MLDS, respectively (Table 3). Absolute agreement between the two MDS derived from the FFQ and $24 \mathrm{~h}$ recalls was determined by the ICC and LOA methods. The ICC was comparable to Pearson's correlations.

Mean agreement was calculated, and Bland-Altman's LOA method was used to determine, in absolute terms,

Table 1 Characteristics of the validation study participants and the REGICOR cohort study participants

\begin{tabular}{|c|c|c|c|c|c|}
\hline & \multicolumn{2}{|c|}{ REGICOR cohort ( $n$ 6215) } & \multicolumn{2}{|c|}{ Validation study ( $n$ 107) } & \multirow[b]{2}{*}{$P$} \\
\hline & $\begin{array}{c}\text { Mean or } \\
\text { median }\end{array}$ & $\begin{array}{l}\text { SD } 95 \% \mathrm{Cl} \text { or } \\
\text { P25 and P75 }\end{array}$ & $\begin{array}{l}\text { Mean or } \\
\text { median }\end{array}$ & $\begin{array}{l}\text { SD } 95 \% \mathrm{Cl} \text { or } \\
\text { P25 and P75 }\end{array}$ & \\
\hline Men (\%) & $52 \cdot 5$ & $51 \cdot 3,53 \cdot 7$ & $48 \cdot 6$ & $39 \cdot 1,58 \cdot 1$ & 0.423 \\
\hline Age (years)* & $56 \cdot 6$ & $12 \cdot 6$ & $58 \cdot 4$ & $12 \cdot 1$ & $0 \cdot 139$ \\
\hline BMI $\left(\mathrm{kg} / \mathrm{m}^{2}\right)^{\star}$ & $27 \cdot 3$ & 4.5 & $27 \cdot 6$ & $4 \cdot 2$ & 0.509 \\
\hline Current smokers (\%) & $22 \cdot 4$ & $21 \cdot 4,23 \cdot 4$ & $16 \cdot 1$ & $8 \cdot 9,23 \cdot 1$ & $0 \cdot 119$ \\
\hline Alcohol intake $(\mathrm{g} / \mathrm{d})$ & $3 \cdot 1$ & $0 \cdot 0,11 \cdot 3$ & $3 \cdot 6$ & $0 \cdot 7,11 \cdot 3$ & 0.446 \\
\hline LTPA $($ MET $\times \min / d)$ & 314 & 119,402 & 327 & 159,426 & $0 \cdot 128$ \\
\hline Education higher than primary school (\%) & $50 \cdot 0$ & $48 \cdot 8,51 \cdot 3$ & $62 \cdot 6$ & $53 \cdot 1,72 \cdot 1$ & 0.010 \\
\hline
\end{tabular}

P25, 25th percentile; P75, 75th percentile; LTPA, leisure-time physical activity; MET, metabolic equivalent of task.

Categorical variables are presented as relative frequencies $(95 \% \mathrm{Cl})$; continuous variables are presented as mean or median (sD or P25 and P75). Differences in continuous variables were compared using the Student $t$ test or the Mann-Whitney $U$ test. Categorical variables were tested using the $\chi^{2}$ goodness-of-fit test. *Data are presented as mean and SD. 
Table 2 Food intake, Spearman's correlation coefficients and agreement between food intake estimates between the FFQ and $24 \mathrm{~h}$ recalls

\begin{tabular}{|c|c|c|c|c|c|c|c|c|c|}
\hline & \multicolumn{3}{|c|}{ FFQ } & \multicolumn{3}{|c|}{$24 \mathrm{~h}$ recalls } & \multirow{2}{*}{$\begin{array}{l}\text { Spearman's } \\
\text { correlations }^{*}\end{array}$} & \multirow{2}{*}{$\frac{\text { Same tertile }}{\%}$} & \multirow{2}{*}{$\frac{\text { Opposite tertile }}{\%}$} \\
\hline & Median & P25 & P75 & Median & P25 & P75 & & & \\
\hline Vegetables (g/4·18MJ) & 231 & 148 & 326 & 78 & 55 & 110 & 0.55 & $56 \cdot 1$ & $10 \cdot 2$ \\
\hline Fruit $(\mathrm{g} / 4 \cdot 18 \mathrm{MJ})$ & 119 & 66 & 184 & 170 & 83 & 234 & $0 \cdot 28$ & $41 \cdot 1$ & $12 \cdot 1$ \\
\hline Nuts (g/4·18 MJ) & $4 \cdot 7$ & $1 \cdot 6$ & $9 \cdot 9$ & $1 \cdot 1$ & 0.0 & $3 \cdot 7$ & $0 \cdot 36$ & $46 \cdot 7$ & $15 \cdot 9$ \\
\hline Meat (g/4·18MJ) & 63 & 42 & 79 & 48 & 35 & 64 & $0 \cdot 26$ & $33 \cdot 6$ & $15 \cdot 9$ \\
\hline Fish $(\mathrm{g} / 4 \cdot 18 \mathrm{MJ})$ & 34 & 22 & 47 & 25 & 14 & 36 & $0 \cdot 19$ & $39 \cdot 3$ & $17 \cdot 8$ \\
\hline Cereals (g/4·18MJ) & 67 & 51 & 93 & 124 & 86 & 153 & $0 \cdot 37$ & $49 \cdot 5$ & $11 \cdot 2$ \\
\hline Legumes (g/4·18 MJ) & 25 & 17 & 35 & 13 & 0 & 29 & $0 \cdot 25$ & $36 \cdot 4$ & $16 \cdot 8$ \\
\hline Dairy $(g / 4 \cdot 18 \mathrm{MJ})$ & 102 & 38 & 165 & 94 & 54 & 136 & $0 \cdot 38$ & $47 \cdot 7$ & $11 \cdot 2$ \\
\hline Olive oil (g/4·18 MJ) & 12 & 8 & 18 & 6 & 3 & 8 & $0 \cdot 33$ & $47 \cdot 6$ & $16 \cdot 8$ \\
\hline Fruit and nuts $(\mathrm{g} / 4 \cdot 18 \mathrm{MJ})$ & 123 & 70 & 186 & 171 & 85 & 239 & $0 \cdot 31$ & $39 \cdot 3$ & $12 \cdot 1$ \\
\hline Red wine $(\mathrm{ml}) \dagger$ & $6 \cdot 7$ & 0 & $50 \cdot 0$ & $10 \cdot 0$ & 0.0 & $45 \cdot 4$ & 0.69 & $73 \cdot 8$ & $0 \cdot 0$ \\
\hline Fast food $(\mathrm{g} / 4 \cdot 18 \mathrm{MJ})$ & $6 \cdot 3$ & 0 & $35 \cdot 3$ & $3 \cdot 8$ & $0 \cdot 0$ & $10 \cdot 6$ & 0.41 & $48 \cdot 7$ & $11 \cdot 3$ \\
\hline Added sugar (g/4·18 MJ) & 0 & 0 & 3.5 & $1 \cdot 1$ & $0 \cdot 2$ & $4 \cdot 4$ & $0 \cdot 67$ & $67 \cdot 6$ & $7 \cdot 6$ \\
\hline Pastry $(\mathrm{g} / 4 \cdot 18 \mathrm{MJ})$ & $1 \cdot 4$ & 0 & $4 \cdot 5$ & $2 \cdot 4$ & 0 & $6 \cdot 3$ & 0.37 & $45 \cdot 8$ & $15 \cdot 6$ \\
\hline Soft drinks (ml/4·18 MJ)‡ & 0 & 0 & $11 \cdot 3$ & $11 \cdot 5$ & 0 & $35 \cdot 3$ & $0 \cdot 32$ & $56 \cdot 1$ & $14 \cdot 9$ \\
\hline Low-fat dairy products $(\mathrm{g} / 4 \cdot 18 \mathrm{MJ})$ & 67 & 0 & 124 & 23 & 0 & 65 & 0.56 & $54 \cdot 2$ & $8 \cdot 4$ \\
\hline Mean & - & - & - & - & - & - & $0 \cdot 39$ & $48 \cdot 4$ & $12 \cdot 4$ \\
\hline
\end{tabular}

P25, 25th percentile; P75, 75th percentile

*Spearman's correlation coefficients between food intake obtained through the FFQ and $24 \mathrm{~h}$ recalls.

tFor cross-classification categorized into $0 \mathrm{ml}, 0 \cdot 1-100 \mathrm{ml}$ and $>100 \mathrm{ml}$ of red wine consumption.

‡Sugar-sweetened carbonated beverages.

Table 3 Mean, correlation coefficients, between-method agreement and limits of agreement between the FFQ and the $24 \mathrm{~h}-\mathrm{R}$

\begin{tabular}{|c|c|c|c|c|}
\hline & \multicolumn{2}{|c|}{ Modified Mediterranean diet score } & \multicolumn{2}{|c|}{ Mediterranean-like diet score } \\
\hline & Energy unadjusted & Energy adjusted & Energy unadjusted & Energy adjusted \\
\hline \multicolumn{5}{|l|}{ FFQ } \\
\hline Mean & $20 \cdot 00$ & $20 \cdot 11$ & $26 \cdot 07$ & $26 \cdot 01$ \\
\hline SD & $2 \cdot 92$ & $2 \cdot 77$ & $3 \cdot 73$ & $3 \cdot 84$ \\
\hline \multicolumn{5}{|l|}{$24 \mathrm{~h}-\mathrm{R}$} \\
\hline Mean & $19 \cdot 99$ & $20 \cdot 00$ & $26 \cdot 44$ & $26 \cdot 61$ \\
\hline SD & $2 \cdot 83$ & $2 \cdot 92$ & $3 \cdot 68$ & $3 \cdot 84$ \\
\hline Mean difference & 0.01 & $0 \cdot 11$ & -0.38 & -0.61 \\
\hline $95 \% \mathrm{Cl}$ & $-0.68,0.70$ & $-0.45,0.67$ & $-1 \cdot 11,0 \cdot 34$ & $-1.25,0.04$ \\
\hline Mean agreement $(\%)^{\star}$ & 101 & 102 & 99 & 99 \\
\hline $95 \% \mathrm{Cl}$ & 98,105 & 99,105 & 97,102 & 96,101 \\
\hline LOA (\%)† & 64,139 & 69,134 & 70,129 & 72,125 \\
\hline$r(\mathrm{FFQ} v .24 \mathrm{~h}-\mathrm{R}) \ddagger$ & 0.33 & 0.48 & 0.42 & 0.62 \\
\hline ICC (FFQ v. 24h-R) & 0.33 & 0.48 & 0.41 & 0.61 \\
\hline
\end{tabular}

$24 \mathrm{~h}-\mathrm{R}, 24 \mathrm{~h}$ recalls; LOA, limits of agreement; ICC, intraclass correlation coefficient.

${ }^{*}$ Mean agreement expressed as (FFQ/24h-R) $\times 100$ and $95 \% \mathrm{Cl}$ of mean agreement.

$+95 \%$ limits of agreement.

‡Pearson's correlation coefficient.

the extent of differences between the scores derived from the FFQ and those derived from $24 \mathrm{~h}$ recalls. There was little difference in mean agreement, or in LOA, between energy-adjusted $v$. unadjusted MDS and MLDS (Table 3). The mean percentage of agreement was close to 100 for all measures, with lower LOA above $50 \%$ and upper limits well below $200 \%$ for both indices. Moreover, for both energy-adjusted and non-adjusted scores of both indices, agreement did not vary with the magnitude of ratings (Fig. 1), indicating no proportional bias.

To analyse construct validity, we hypothesized a priori relationships between higher scores and more favourable intake profiles for fifteen nutrients. We found that, of these fifteen nutrients, $73.3 \%$ and $86.7 \%$ of the $24 \mathrm{~h}$ recallderived intake estimates were associated significantly and in the anticipated direction with tertiles of the FFQ-derived mMDS and MLDS, respectively (Table 4).

\section{Discussion}

The present study determined the concurrent and construct validity of two variants of the MDS. Compared with data derived from multiple $24 \mathrm{~h}$ recalls spanning a 12-month period, the FFQ showed an adequate capacity to rank participants on the basis of two MDS, the MMDS and the MLDS. LOA was in a reasonable range for both scores. Furthermore, our results indicate sound evidence for construct validity, particularly for the MLDS score, which incorporated several modifications into the traditional MDS. 

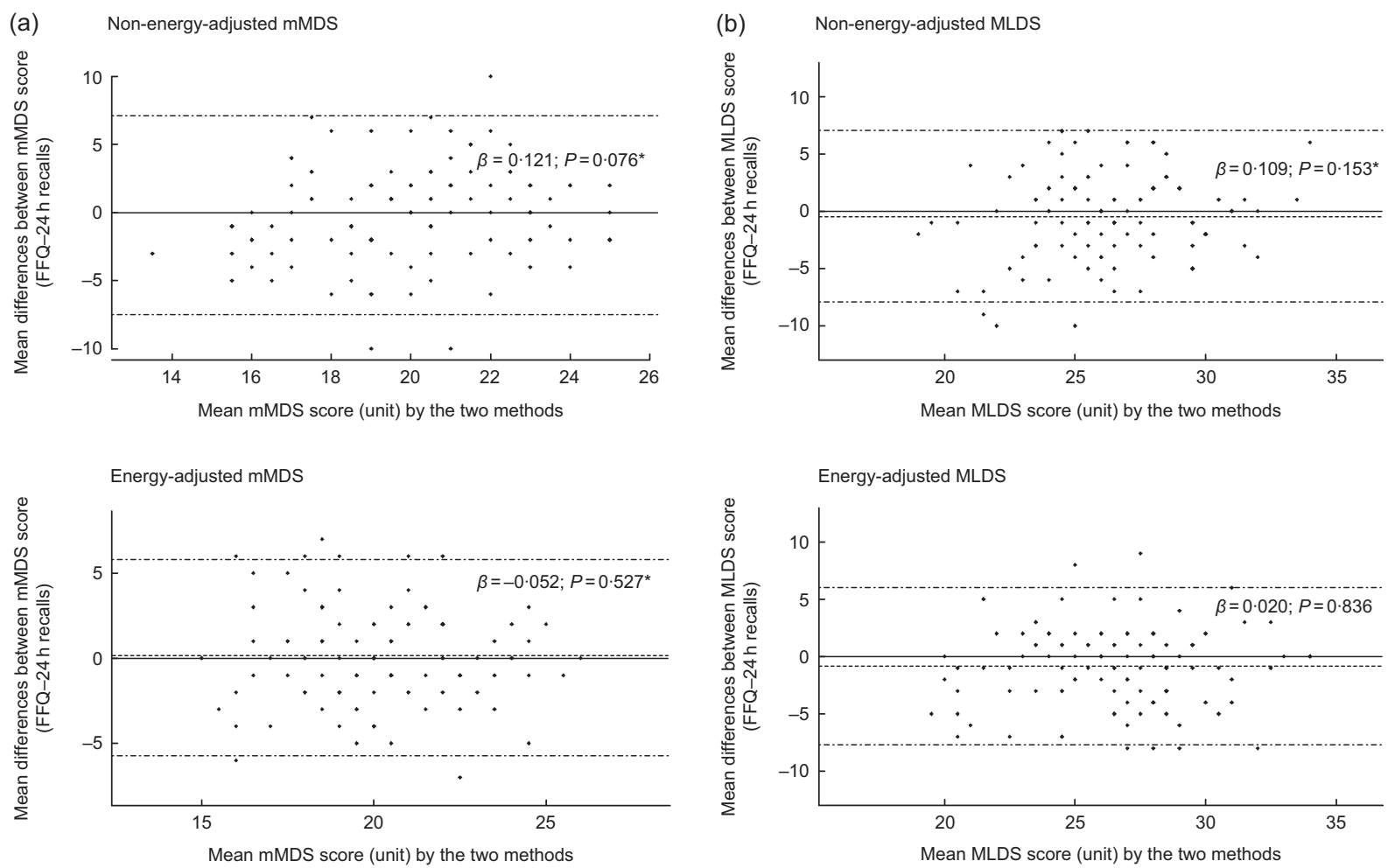

Fig. 1 Mean differences of the modified Mediterranean diet score (mMDS; a) and the Mediterranean-like diet score (MLDS; b) derived from the FFQ and $24 \mathrm{~h}$ recalls. Lines are mean differences (----) and upper and lower $95 \%$ limits of agreement (--.--); *regression coefficient and statistical significance of the slope from the linear regression of the mean of the methods against the difference between methods

Table 4 Nutrient intake by quartile of energy-adjusted dietary scores

\begin{tabular}{|c|c|c|c|c|c|c|c|c|c|c|}
\hline & \multicolumn{5}{|c|}{ Modified Mediterranean diet score } & \multicolumn{5}{|c|}{ Mediterranean-like diet score } \\
\hline & \multicolumn{2}{|c|}{ 1st quartile ( $n$ 32) } & \multicolumn{2}{|c|}{ 4th quartile ( $n 21)$} & \multirow[b]{2}{*}{$P^{\star}$} & \multicolumn{2}{|c|}{ 1st quartile (n 21) } & \multicolumn{2}{|c|}{ 4th quartile ( $n 25)$} & \multirow[b]{2}{*}{$P^{*}$} \\
\hline & Mean & SD & Mean & SD & & Mean & SD & Mean & SD & \\
\hline Saturated/unsaturated fat & 0.52 & $0 \cdot 10$ & 0.45 & 0.07 & 0.020 & 0.51 & 0.09 & 0.44 & 0.07 & 0.015 \\
\hline Cholesterol $(\mathrm{mg} / \mathrm{d})$ & 156 & $34 \cdot 5$ & 151 & $29 \cdot 5$ & 0.293 & 167 & $41 \cdot 3$ & 160 & $47 \cdot 1$ & 0.666 \\
\hline Trans fatty acids (g/4·18 MJ) & 0.49 & 0.36 & 0.45 & 0.35 & 0.405 & $0 \cdot 71$ & 0.47 & 0.39 & $0 \cdot 37$ & 0.009 \\
\hline Fibre $(g / 4 \cdot 18 \mathrm{MJ})$ & $8 \cdot 1$ & $2 \cdot 5$ & $10 \cdot 2$ & $2 \cdot 9$ & 0.009 & 6.5 & $2 \cdot 2$ & $10 \cdot 6$ & $2 \cdot 8$ & $<0.001$ \\
\hline $\mathrm{Na}(\mathrm{mg} / 4 \cdot 18 \mathrm{MJ})$ & 1315 & 235 & 13475 & 417 & $0 \cdot 775$ & 1320 & 260 & 1407 & 412 & 0.392 \\
\hline $\mathrm{K}(\mathrm{mg} / 4 \cdot 18 \mathrm{MJ})$ & 1405 & 272 & 1647 & 290 & 0.004 & 1268 & 247 & 1699 & 365 & $<0.001$ \\
\hline $\mathrm{Mg}(\mathrm{mg} / 4 \cdot 18 \mathrm{MJ})$ & 127 & $22 \cdot 9$ & 146 & $24 \cdot 1$ & 0.002 & 116 & $16 \cdot 1$ & 152 & $29 \cdot 5$ & $<0.001$ \\
\hline $\mathrm{Ca}(\mathrm{mg} / 4 \cdot 18 \mathrm{MJ})$ & 452 & 125 & 458 & $91 \cdot 1$ & 0.650 & 388 & $96 \cdot 4$ & 501 & 103 & $<0.001$ \\
\hline Vitamin C (mg/4·18 MJ) & $42 \cdot 1$ & $20 \cdot 6$ & $72 \cdot 5$ & $26 \cdot 4$ & 0.001 & $34 \cdot 2$ & $20 \cdot 0$ & $69 \cdot 7$ & $28 \cdot 9$ & $<0.001$ \\
\hline Vitamin E (mg/4·18 MJ) & $3 \cdot 0$ & $0 \cdot 8$ & $3 \cdot 7$ & 0.9 & 0.004 & $2 \cdot 9$ & 0.7 & 3.9 & $1 \cdot 1$ & $<0.001$ \\
\hline Folic acid $(\mu \mathrm{g} / 4 \cdot 18 \mathrm{MJ})$ & 112 & $28 \cdot 4$ & 141 & $28 \cdot 8$ & 0.002 & $96 \cdot 8$ & $25 \cdot 5$ & 148 & $37 \cdot 7$ & $<0.001$ \\
\hline Carotene (mg/4·18 MJ) & $12 \cdot 3$ & $8 \cdot 2$ & $18 \cdot 6$ & $9 \cdot 4$ & 0.034 & $12 \cdot 1$ & $9 \cdot 2$ & $18 \cdot 4$ & $9 \cdot 9$ & 0.015 \\
\hline Lycopene (mg/4·18 MJ) & 0.56 & 0.49 & $1 \cdot 20$ & 0.97 & $<0.001$ & 0.52 & 0.41 & $1 \cdot 21$ & 0.86 & $<0.001$ \\
\hline Phytosterols (mg/4·18 MJ) & $68 \cdot 5$ & $17 \cdot 0$ & $93 \cdot 6$ & $28 \cdot 6$ & $<0.001$ & $66 \cdot 7$ & $19 \cdot 1$ & $93 \cdot 2$ & $28 \cdot 6$ & $<0.001$ \\
\hline Flavonoids (mg/4.18MJ) & $31 \cdot 0$ & $18 \cdot 4$ & $50 \cdot 0$ & $19 \cdot 5$ & 0.003 & $29 \cdot 8$ & $19 \cdot 6$ & $46 \cdot 5$ & $23 \cdot 1$ & 0.011 \\
\hline
\end{tabular}

${ }^{*} P$ for linear trend.

The Mediterranean diet is a healthy eating pattern associated with better health and lower risk of premature mortality $^{(5-9,19-21)}$. Since 1995 , adherence to this dietary pattern has been assessed by FFQ-derived composite scores that include foods that are characteristic of the Mediterranean olive grove areas ${ }^{(4)}$. Although considered an adequate dietary assessment tool for the estimation of overall diet quality, the MDS has varied between studies because of differences in the scoring criteria $^{(4)}$. The present study assessed the validity of a score previously proposed by our group ${ }^{(16)}$. In a further adjustment, we omitted inverse scoring for dairy products, including 
instead low-fat and fat-free (skim) dairy products as healthy foods. We also added fast food, sugar-sweetened carbonated beverages, added sugars and pastry as detrimental foods. Finally, we excluded poultry and rabbit from the meat and sausage food group, scoring these meats separately as a healthy choice. We hypothesized that these changes would increase the accuracy of the construct to measure diet quality. Indeed, overall, both the magnitude of correlations and the proportion of nutrients supporting construct validity were somewhat higher for the modified score than for the original score.

Correlation coefficients for individual food group components of the mMDS and MLDS ranged from $0 \cdot 19$ to $0 \cdot 69$, which is comparable to results from other similar validation studies ${ }^{(10)}$. In addition, we found a reasonable frequency of agreement and gross misclassification of food groups between methods. Both the mMDS and the MLDS were moderately correlated between assessment methods, indicating that the FFQ reasonably ranks participants according to these diet-quality indices. Unfortunately, the limited literature on concurrent validity of dietary indices makes it somewhat difficult to compare our results with those of other studies. However, a few studies have reported correlations of similar magnitude. Newby et l $^{(22)}$ reported good correlations $(r=0 \cdot 66)$ between the Diet Quality Index Revised derived from an FFQ and those from two 1 -week dietary records. Results from $\mathrm{Hu}$ et al. $^{(23)}$ showed the validity of two major dietary patterns derived using principal component analysis from dietary estimates of an FFQ compared with multiple dietary records, with correlations of $0.45-0.58$ for the first FFQ.

Ideally, studies on the validity of an FFQ should include multiple methodological tools to determine validity. This permits an accurate interpretation of the strengths and weaknesses of the instrument and provides insight into possible biases of the dietary assessment method. For this reason we assessed the relative validity of the mMDS and MLDS using cross-classification, correlation coefficients, Bland-Altman plots and the LOA method proposed by Bland and Altman ${ }^{(17)}$. The mean agreement of scores between methods was reasonable in the present study, and LOA was well within the acceptable boundaries of $50 \%$ and $200 \%{ }^{(18)}$. In the present study, the LOA for both scores was in a narrow range compared with levels reported in previous studies for individual nutrients ${ }^{(18,24)}$; for example, the FFQ underand overestimated the dietary recall estimates of the energy-adjusted MLDS by only $28 \%$ and $25 \%$, respectively. Furthermore, Bland-Altman plots showed no significant proportional variations over the range of average ratings for any of the three dietary indices. This means that the errors were not proportional to the ratings.

Construct validity is an additional aspect to be considered in selecting a dietary assessment tool. To address this issue we hypothesized that both of the FFQ-derived dietary quality indices would be positively associated with a favourable nutrient intake profile estimated by $24 \mathrm{~h}$ recalls. Intakes of $\mathrm{K}, \mathrm{Mg}$, folic acid, vitamins $\mathrm{C}$ and $\mathrm{E}$, phytosterols and dietary fibre were positively associated with mMDS ratings. In addition to these nutrients, inclusion in the MLDS of additional detrimental foods such as fast food, added sugars, sugar-sweetened soft drinks and pastry - as well as modification of the dairy component improved the construct validity of the score and yielded a positive association with trans fatty acids and Ca.

An inherent limitation of our study, and of all validation studies using multiple dietary recalls or records as the reference method, is that these methods themselves are not error free. Errors in the two methods may be correlated. However, the measures used in the present study have important strengths, including the use of unannounced recalls spanning a 12 -month period, enabling us to capture seasonal differences in intake.

We conclude that the FFQ accurately allocates participants across the distribution of ratings of the mMDS and MLDS intakes. For both scores, the construct estimates were valid compared with multiple recalls, and the LOA was in a reasonable range with no indication of bias.

\section{Acknowledgements}

The present research was supported by a grant from Fondo Europeo de Desarrollo Regional (FEDER; Grant no. 2FD097-0297-CO2-01) and by parts of grants from Spain's Ministerio de Sanidad y Consumo, Instituto de Salud Carlos III FEDER (Grant no. PI080439), Red HERACLES RD06/ 0009 and by a joint contract of the Instituto de Salud Carlos III and the Health Department of the Catalan Government (Generalitat de Catalunya; CP 03/00115). The CIBERobn is an initiative of the Instituto de Salud Carlos III, Madrid, Spain. The authors have no conflict of interest to declare. A.A.B.-A. conducted the analyses and prepared the manuscript; H.Sc. designed the study; M.A.M., J.M.B.-D., M.A.R.A., C.S., J.M., M.-I.C., H.S., A.L. and H.Sc. provided their expertise in data analysis and in interpretation and discussion of results and made substantial suggestions to the manuscript. All authors read and approved the final manuscript. The authors appreciate the English revision made by Elaine Lilly, PhD (Writers First Aid).

\section{References}

1. Kant AK (2004) Dietary patterns and health outcomes. J Am Diet Assoc 104, 615-635.

2. Waijers PM, Feskens EJM \& Ocké C (2007) A critical review of predefined diet quality scores. Br J Nutr 97, 219-231.

3. Arvaniti F \& Panagiotakos DB (2008) Healthy indexes in public health practice and research: a review. Crit Rev Food Sci Nutr 48, 317-327.

4. Bach A, Serra-Majem L, Carrasco JL et al. (2006) The use of indexes evaluating the adherence to the Mediterranean diet in epidemiological studies: a review. Public Health Nutr 9 , $132-146$. 
5. Trichopoulou A, Costacou T, Bamia C et al. (2003) Adherence to a Mediterranean diet and survival in a Greek population. N Engl J Med 348, 2599-2608.

6. Trichopoulou A, Kouris-Blazos A, Wahlqvist ML et al. (1995) Diet and overall survival in elderly people. BMJ311, $1457-1460$.

7. Martinez-Gonzalez MA, Bes-Rastrollo M, Serra-Majem L et al. (2009) Mediterranean food pattern and the primary prevention of chronic disease: recent developments. Nutr Rev 67, Suppl. 1, S111-S116.

8. Muñoz MA, Fíto M, Marrugat J et al. (2009) Adherence to the Mediterranean diet is associated with better mental and physical health. Br J Nutr 101, 1821-1827.

9. Schroder H (2007) Protective mechanisms of the Mediterranean diet in obesity and type 2 diabetes. J Nutr Biochem 18, 149-160

10. Molag ML, de Vries JH, Ocké MC et al. (2007) Design characteristics of food frequency questionnaires in relation to their validity. Am J Epidemiol 166, 1468-1478.

11. Kristal AR \& Potter JD (2006) Not the time to abandon the food frequency questionnaire: counterpoint. Cancer Epidemiol Biomarkers Prev 15, 1759-1760.

12. Freedman LS, Potischman N, Kipnis V et al. (2006) A comparison of two dietary instruments for evaluating the fat-breast cancer relationship. Int J Epidemiol 35, 1011-1021.

13. Grau M, Subirana I, Elosua R et al. (2007) Trends in cardiovascular risk factor prevalence (1995-2000-2005) in northeastern Spain. Eur J Cardiovasc Prev Rehabil 14, 653-659.

14. Schröder H, Covas MI, Marrugat J et al. (2002) Use of a three-day estimated food record, a 72-hour recall and a food-frequency questionnaire for dietary assessment in a Mediterranean Spanish population. Clin Nutr 20, 429-437.
15. Trichopoulou A, Orfanos P, Norat T et al. (2005) Modified Mediterranean diet and survival: EPIC-elderly prospective cohort study. BMJ 330, 991.

16. Schröder H, Marrugat J, Vila J et al. (2004) Adherence to the traditional Mediterranean diet is inversely associated with body mass index and obesity in a Spanish population. J Nutr 134, 3355-3361.

17. Bland JM \& Altman DG (1986) Statistical methods for assessing agreement between two methods of clinical measurement. Lancet 1, 307-310.

18. Ambrosini GL, van Roosbroeck SA, Mackerras D et al. (2003) The reliability of ten-year dietary recall: implications for cancer research. J Nutr 133, 2663-2668.

19. Scarmeas N, Stern Y, Tang MX et al. (2006) Mediterranean diet and risk of Alzheimer's disease. Ann Neurol 59, 912-921.

20. Buckland G, González CA, Agudo A et al. (2009) Adherence to the Mediterranean diet and risk of coronary heart disease in the Spanish EPIC cohort study. $A m J$ Epidemiol 170, 1518-1529.

21. Benetou V, Trichopoulou A, Orfanos P et al. (2008) Conformity to traditional Mediterranean diet and cancer incidence: the Greek EPIC cohort. Br J Cancer 99, 191-195.

22. Newby PK, Hu FB, Rimm EB et al. (2003) Reproducibility and validity of the Diet Quality Index Revised as assessed by use of a food-frequency questionnaire. Am J Clin Nutr 78, 941-949.

23. Hu FB, Rimm E, Smith-Warner SA et al. (1999) Reproducibility and validity of dietary patterns assessed with a foodfrequency questionnaire. Am J Clin Nutr 69, 243-249.

24. Keogh JB, Lange K \& Syrette J (2010) Comparative analysis of two FFQ. Public Health Nutr 13, 1553-1558. 